doi: https://doi.org/10.15407/dopovidi2018.11.032

УДК 532.546

\title{
В.Л. Поляков
}

Институт гидромеханики НАН Украины, Киев

E-mail: polyakov_igm@list.ru

\section{Моделирование осветления суспензии при усиленном влиянии нерегулируемой скорости фильтрования на межфазный массообмен}

\author{
Представлено членом-корреспондентом НАН Украины А.Я. Олейником
}

Сформулирована математическая задача осветления суспензии на скорых фильтрах с накопителем при нерегулируемой скорости, с которой линейным образом связаны массообменные коэффициенты. Получено эффективное аналитико-численное решение задачи, позволяющее надежно прогнозировать изменения характеристик фильтрования со временем и по высоте с учетом сопротивления отводящих коммуникащий, обосновывать рациональные технологические параметры и в первую очередь длительность фильтрочикла. На характерных примерах решение иллюстрируется расчетами концентрации взвеси в фильтрате, скорости фильтрования и технологических времен.

Ключевые слова: фильтрование, суспензия, нерегулируемая скорость, технология, массообмен, фильтр.

Фильтрование суспензий через пористые загрузки очистных фильтров на практике осуществляется как с регулируемой (постоянной), так и с нерегулируемой скоростью. Теория фильтрования с постоянной скоростью в настоящее время разработана основательно (см. например [1, 2]), что объясняется и высокими практическими потребностями в ней, и относительной простотой ее базовых математических моделей. Такие модели допускают поэтапную реализацию составляющих их блоков, что значительно облегчает получение аналитических решений. Намного сложнее моделировать комплексный технологический процесс в отсутствии регуляторов скорости. Тем не менее в последнее время проведены серьезные исследования фильтрования с нерегулируемой скоростью, используя и аналитические, и численные методы [3-7]. Однако полученные таким образом решения являются приближенными и в связи с разнообразием условий фильтрования постоянно возникает вопрос о достоверности результатов расчетов, проведенных с их помощью. Поэтому важное значение приобретают эталонные решения, которые бы позволяли, с одной стороны, оперативно контролировать процесс вычислений, а с другой - были бы полезными при проектировании и эксплуатации фильтровальных сооружений. В данной работе построено одно из таких решений в аналитической форме, причем удалось избежать каких-либо упрощений и при по-

(C) В.Л. Поляков, 2018 
становке исходной задачи, и в ходе ее реализации. Однако здесь приходится на одном из промежуточных этапов расчетов решать численно обыкновенное дифференциальное уравнение первого порядка, что просто сделать с любыми наперед заданными дискретом и точностью, привлекая современные пакеты программ математического анализа (MathCad, Matlab и пр.). Поэтому указанное решение гарантирует высокую точность вычислений и может рассматриваться как эталонное.

Принципиальное значение при формальном описании фильтрования в целом и конкретно обмена дисперсными частицами между жидкой и твердой фазами имеет учет влияния на него скорости фильтрования $V$. Согласно рекомендации $[8,9]$, для функциональных коэффициентов скорости прилипания $\alpha$ и отрыва $\beta$ частиц суспензии оправдано принимать степенные аппроксимационные выражения

$$
\alpha=\alpha_{V} V^{l}, \beta=\beta_{V} V^{q},
$$

где $\alpha_{V}, \beta_{V}-$ соответствующие приведенные (постоянные) коэффициенты; $l, q-$ показатели, которые могут определяться теоретическим или экспериментальным путем.

Работу водоочистных фильтров удается интенсифицировать благодаря использованию в его конструкции накопителя (стакана), в котором над загрузкой накапливается избыток суспензии. Обычно суспензия подается на фильтр с расходом $Q_{i n}$, который превышает его первоначальную пропускную способность. Таким образом, часть ее задерживается в стакане, а часть поступает в загрузку. При этом и также вследствие прогрессирующего заиления фильтрующего материала уровень суспензии над загрузкой неуклонно повышается.

Соответствующая математическая модель включает кроме двух традиционных (осветлительный, фильтрационный) блоков и дополнительный (гидравлический) блок. В целом модель содержит систему уравнений [10]

$$
\begin{aligned}
& V(t) \frac{\partial C}{\partial z}+\frac{\partial S}{\partial t}=0 ; \\
& \frac{\partial S}{\partial t}=\alpha_{V} V(t) C-\beta_{V} V(t) S ; \\
& V(t)=-k\left(S_{s}\right) \frac{\partial h}{\partial z} ; \\
& k\left(S_{s}\right)=k_{0} f_{k}\left(S_{s}\right) ; \\
& S_{s}(S)=\gamma(S) S ; \\
& \omega \frac{d H}{d t}=Q_{i n}-\omega V(t)
\end{aligned}
$$

и оператор граничных и начальных условий (координатная ось направлена вниз от поверхности загрузки)

$$
\begin{array}{ll}
z=0, & C=C_{0} ; \\
z=L, & h=H_{\text {out }}+R \omega^{2} V^{2}(t) ; \\
t=0, & H=H^{0} ; \quad S=0 .
\end{array}
$$


Здесь $C, S$ - объемные концентрации взвешенных и осажденных частиц суспензии; $k$, $k_{0}$ - коэффициент фильтрации заиленного и чистого фильтрующего материала; $S_{s}-$ объемная концентрация осадка; $h$ - пьезометрический напор; $f_{k}\left(S_{s}\right)-$ эмпирическая функция, характеризующая ухудшение фильтрационных свойств загрузки по мере накопления в ней осадка; $\boldsymbol{\gamma}$ - отношение объемных концентраций осадка и твердых частиц в нем, характеризующее содержание в первом связанной воды; $\omega, L-$ площадь поверхности и высота загрузки; $H_{\text {out }}$ - пьезометрический напор в коллекторе фильтрата; $R$ - гидравлическое сопротивление отводящих коммуникаций; $C_{0}, H^{0}-$ исходные концентрация взвеси и уровень в стакане.

Существенным ограничением, принятым в данной постановке, является единственность значений $l$ и $q(l=q=1)$. Его обоснованность подтверждается результатами и теоретических, и экспериментальных исследований $[11,12]$. Результаты теоретических исследований обобщаются благодаря введению следующих безразмерных переменных и параметров: $\bar{C}=C / C_{0}, \bar{S}=S /\left(n_{0} C_{0}\right), \bar{z}=z / L, \bar{t}=k_{0} t /\left(n_{0} L\right), \bar{V}=V / k_{0}, \bar{h}=\left(h-H_{\text {out }}\right) / L, \bar{\alpha}_{V}=L \alpha_{V}$, $\bar{\beta}_{V}=n_{0} L \beta_{V}, \bar{k}=k / k_{0}, \bar{\gamma}=\gamma C_{0}, \bar{R}=\omega^{2} k_{0}^{2} R / L, \bar{Q}_{i n}=Q_{i n} /\left(\omega k_{0}\right)$, где $n_{0}$ - пористость чистой загрузки. Тогда задача (2)-(10) после введения замены

$$
\tau=\int_{0}^{\bar{t}} \bar{V}(\varsigma) d \varsigma
$$

преобразуется к такому виду:

$$
\begin{aligned}
& \frac{\partial \bar{C}}{\partial \bar{z}}+\frac{\partial \bar{S}}{\partial \tau}=0 ; \\
& \frac{\partial \bar{S}}{\partial \tau}=\bar{\alpha}_{V} \bar{C}-\bar{\beta}_{V} \bar{S} ; \\
& \bar{V}(\bar{t})=-\bar{k}(\bar{S}) \frac{\partial \bar{h}}{\partial \bar{z}} ; \\
& \bar{k}(\bar{S})=f_{k}(\bar{S}) ; \\
& \frac{d \bar{H}}{d \bar{t}}=n_{0}\left[\bar{Q}_{i n}-\bar{V}(\bar{t})\right] ; \\
& \bar{z}=0, \quad \bar{C}=1 ; \\
& \bar{z}=1, \quad \bar{h}=\bar{R} \bar{V}^{2}(\bar{t}) ; \\
& \bar{t}=0, \quad \bar{S}=0 ; \quad \bar{H}=\bar{H}^{0} .
\end{aligned}
$$

Решение осветлительного блока, включающего уравнения (11), (12) и условия (16), (18), несложно получить операционным методом и тогда искомые концентрации выражаются следующими строгими зависимостями:

$$
\bar{C}(\bar{z}, \tau)=e^{-\bar{\alpha}_{V} \bar{z}}\left[e^{-\bar{\beta}_{V} \tau} I_{0}\left(2 \sqrt{\bar{\alpha}_{V} \bar{\beta}_{V} \bar{z} \tau}\right)+\bar{\beta}_{V} \int_{0}^{\tau} e^{-\bar{\beta}_{V} \varsigma} I_{0}\left(2 \sqrt{\bar{\alpha}_{V} \bar{\beta}_{V} \bar{z} \varsigma}\right) d \varsigma\right],
$$




$$
\bar{S}(\bar{z}, \tau)=\bar{\alpha}_{V} e^{-\bar{\alpha}_{V} \bar{z}} \int_{0}^{\tau} e^{-\bar{\beta}_{V} \varsigma} I_{0}\left(2 \sqrt{\bar{\alpha}_{V} \bar{\beta}_{V} \bar{z} \varsigma}\right) d \zeta .
$$

В результате решения фильтрационного блока, содержащего уравнения (13), (14) и условие (17), найдено выражение для скорости фильтрования

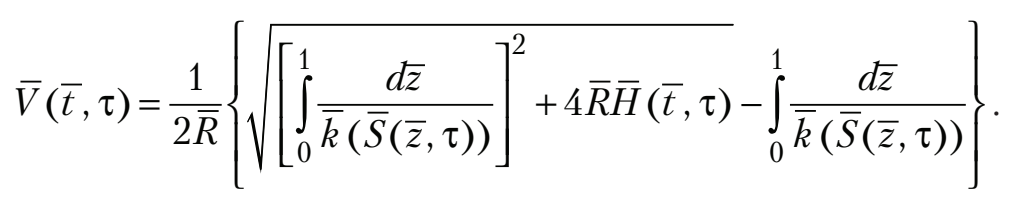

При этом, как следует из гидравлического блока, состоящего из уравнения (15) и условий (17), (18), подъем уровня в стакане описывается зависимостью

$$
\bar{H}(\bar{t}, \tau)=\bar{H}^{0}+n_{0}\left(\bar{Q}_{i n} \bar{t}-\tau\right) .
$$

Из (21) с учетом (31) и интегральной связи между $\tau$ и $\bar{V}$ следует задача Коши относительно функции $\tau(\bar{t})$

$$
\begin{aligned}
& \frac{d \tau}{d \bar{t}}=\frac{1}{2 \bar{R}}\left\{\sqrt{\left.\left[\int_{0}^{1} \frac{d \bar{z}}{\bar{k}(\bar{S}(\bar{z}, \tau))}\right]^{2}+4 \bar{R}\left[\bar{H}^{0}+n_{0}\left(\bar{Q}_{i n} \bar{t}-\tau\right)\right]-\int_{0}^{1} \frac{d \bar{z}}{\bar{k}(\bar{S}(\bar{z}, \tau))}\right\} ;}\right. \\
& \bar{t}=0, \quad \tau=0 .
\end{aligned}
$$

Задача (23), (24) легко решается численно, если привлечь известные пакеты программ математического анализа, например MathCad, Matlab и пр. После нахождения удовлетворяющей ей совокупности пар значений $\tau, \bar{t}$ дальше просто с таким же дискретом по $\bar{t}$ вычислять искомые характеристики $\bar{S}, \bar{C}, \bar{V}, \bar{H}$ как функции от $\bar{t}$. Следует заметить, что интерес для практики также представляет сама зависимость $\tau(\bar{t})$, поскольку $\tau$ по существу выражает относительный удельный (на единицу площади поверхности загрузки) объем суспензии, обработанной к расчетному моменту времени.

В частном случае совершенных в гидравлическом отношении отводящих коммуникаций $(\bar{R}=0)$ из $(21),(23)$, воспользовавшись правилом Лопиталя, несложно получить следующую пару уравнений:

$$
\frac{d \tau}{d \bar{t}}=\left[\bar{H}^{0}+n_{0}\left(\bar{Q}_{i n} \bar{t}-\tau\right)\right]\left[\int_{0}^{1} \frac{d \bar{z}}{\bar{k}(\bar{S}(\bar{z}, \tau))}\right]^{-1}=\bar{V}(\bar{t}, \tau) .
$$

Их последовательное использование дает возможность определить сначала связь между $\tau$ и $\bar{t}$, а затем и вычислить скорость $\bar{V}$.

Ценность для практики представленного выше аналитико-численного решения задачи фильтрования слабоконцентрированной суспензии с нерегулируемой скоростью прежде всего заключается в возможности его использования для надежного обоснования технологических и конструктивных параметров, главным образом, длительности фильтроцикла $\bar{t}_{f}$ [13]. В системе ограничений, накладываемых при этом на комплексный технологический 

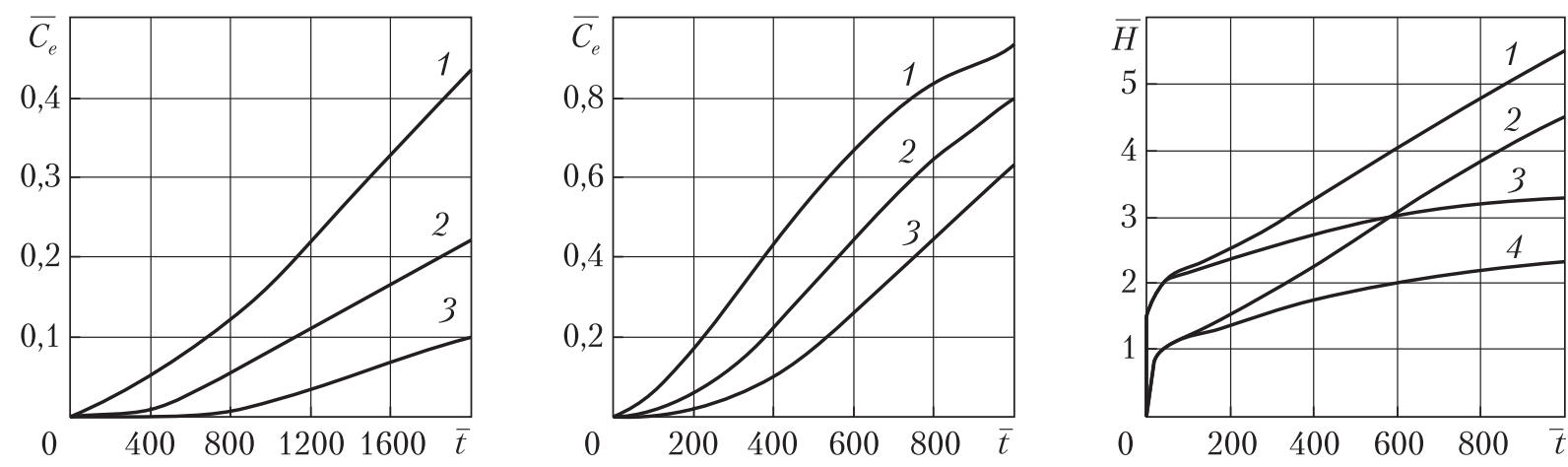

Puc. 1. Рост относительной объемной концентрации взвешенного вещества в фильтрате со временем $\left(\bar{Q}_{i n}=0,2\right): 1-\bar{\alpha}_{V}=5 ; 2-\bar{\alpha}_{V}=7 ; 3-\bar{\alpha}_{V}=9$

Puc. 2. Рост относительной объемной концентрации взвешенного вещества в фильтрате со временем $\left(\bar{Q}_{i n}=1\right): 1-\bar{\alpha}_{V}=5 ; 2-\bar{\alpha}_{V}=7 ; 3-\bar{\alpha}_{V}=9$

$\boldsymbol{P u c . ~ 3 . ~ П о д ъ е м ~ о т н о с и т е л ь н о г о ~ у р о в н я ~ с у с п е н з и и ~ н а д ~ з а и л я е м о и ̆ ~ з а г р у з к о и ̆ ~ с о ~ в р е м е н е м : ~} 1,2-\overline{\boldsymbol{\alpha}}_{V}=9$; $3,4-\bar{\alpha}_{V}=5 ; 1,3-\bar{R}=1 ; 2,4-\bar{R}=0$

процесс, особую роль играет требование обеспечивать только высокое качество фильтрата. Другими словами, концентрация взвеси в фильтрате не должна превышать нормативного значения $C_{*}$. Время повышения выходной концентрации до значения $C_{*}$, так называемое время защитного действия загрузки $t_{p}$, определяется подбором из уравнения (19) при $\bar{z}=1$ с учетом (23), (24). Со временем скорость фильтрования должна снижаться. Второе технологическое время $t_{V}$, а именно время снижения скорости $V$ до минимально допускаемой из экономических соображений $V_{*}$, предлагается вычислять из (21) и опять с учетом (23), (24). Наконец, чтобы не осложнять эксплуатацию фильтра с накопителем суспензии, нежелательно допускать переполнение его стакана. Время $t_{H}$, за которое он успеет наполниться, также целесообразно использовать в качестве одного из временных ограничителей при установлении ключевого технологического параметра - длительности $t_{f}$. После определения значений всех технологических времен $\left(t_{p}, t_{V}, t_{H}\right)$ логично отождествлять $t_{f}$ с наименьшим из них, так что

$$
\bar{t}_{f}=\min \left(t_{p}, t_{V}, t_{H}\right) .
$$

Выведенные аналитическим путем зависимости для относительных величин выходной концентрации, уровня над загрузкой и скорости фильтрования иллюстрируются на ряде примеров с характерными исходными данными. Информация о модельных параметрах подбиралась из литературных источников, а также привлекались данные экспериментальных исследований, проводившихся в Киевском национальном университете строительства и архитектуры на протяжении 2015-2017 гг. Значения некоторых из них изменялись в ходе вычислений непрерывно $\left(\bar{\alpha}_{V}\right)$ или дискретно $\left(\bar{\alpha}_{V}, \bar{Q}_{i n}, \bar{\gamma}, \bar{R}\right)$. Значения остальных модельных и критериальных параметров зафиксированы ( $\bar{\beta}_{V}=0,01, n_{0}=0,47, \bar{H}=0, \bar{C}_{*}=0,1$, $\left.\bar{H}_{*}=4\right)$. Производительность фильтра с накопителем прежде всего определяется расходом поступающей на фильтр суспензии, для которого выбраны три существенно различающихся значения $(0,2,0,5$ и 1). При большем из них подача намного превосходит пропускную 
Puc. 4. Зависимости $\bar{t}_{p}\left(\bar{\alpha}_{V}\right), \bar{t}_{H}\left(\bar{\alpha}_{V}\right), \bar{t}_{V}\left(\bar{\alpha}_{V}\right): 1,2,7-\bar{t}_{H}$; $3-5-\bar{t}_{p} ; 6-\bar{t}_{V} ; 1,5-$ пример $3 ; 2,4-$ пример 1;3, 6, $7-$ пример $2 ; \bar{t}_{f}\left(\bar{\alpha}_{V}\right)-$ сплошные линии

способность фильтра. Как следствие, значительно быстрее накапливается в его стакане суспензия и растет входной напор, что тем самым интенсифицирует фильтрационный процесс, а вместе с ним усиливает образование осадка. При меньшем же значении $\bar{Q}_{i n}$ подъем уровня над загрузкой фактически обусловлен лишь ее заилением и поэтому его темп оказывается меньшим. При этом содержание взвешенного вещества в фильтрате сохраняется низким намного

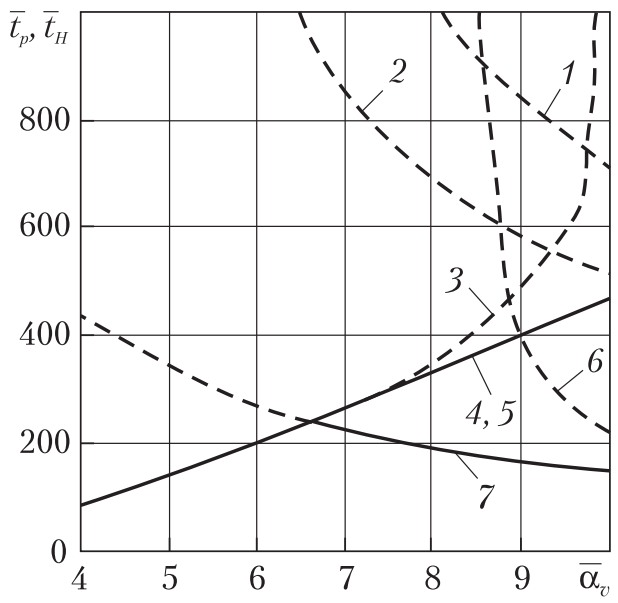
дольше (рис.1) особенно при его усиленной сорбции (кривая 3). Здесь вычисления $\bar{C}_{e}$ выполняли по формуле (19) с учетом (23), (24). Фильтрационный процесс резко интенсифицируется при $\bar{Q}_{i n}=1$, а значит усиливается проскок взвеси через слой загрузки. И, кроме того, со временем защитный ресурс загрузки ускоренно исчерпывается (рис.2). Поэтому необходим особо тщательный контроль за $\bar{C}_{e}$, что обеспечивает (19).

Уровень суспензии в стакане фильтра поднимается неравномерно в еще большей степени (рис.3). На начальном этапе осветления происходил почти скачкообразный прирост величины $\bar{H}$, который затем сменяется плавным увеличением. Расчет $\bar{H}$ выполнялся по (22), причем $\tau$ и $\bar{t}$ связаны, опять-таки, согласно (23), (24). Для оценки значимости сопротивления отводящих коммуникаций помимо $\bar{R}=1$ также полагалось $\bar{R}=0$. Очевидно, что гипотетические изменения сорбционных свойств загрузки ( $\bar{\alpha}_{V}=5$ и 9$)$ и сопротивления коммуникаций $-(\bar{R}=1$ и 1$)$ могут существенно отразиться на динамике уровня.

И в заключение выполнен технологический анализ при непрерывном варьировании сорбционных свойств загрузки в широких пределах, а именно, коэффициент $\bar{\alpha}_{V}$ формально изменялся от 4 до 10. Данные расчетов технологических времен при $\bar{Q}_{i n}=1, \bar{C}_{*}=0,1, \bar{H}_{*}=4$ для трех примеров приведены на рис.4. В первом примере принималось $\bar{\gamma}=0,0005, \bar{R}=1$; во втором $-\bar{\gamma}=0,001, \bar{R}=1$; в-третьем $-\bar{\gamma}=0,0005, \bar{R}=0$. Максимальное расчетное время составило 1000 , что ориентировочно отвечает 3 суткам и намного превосходит реальную длительность фильтроцикла. Выяснилось, что влияние на фильтрационный процесс растущего поверхностного слоя суспензии и осадка, накапливающегося в порах слоя умеренно и хорошо сорбирующего материала, длительное время взаимно компенсируется. Как следствие, скорость фильтрования и однозначно связанная с ней производительность фильтра долго остаются стабильными и достаточно большими. В подобных стандартных ситуациях причиной остановки фильтра должны стать или чрезмерный проскок взвешенного вещества через слой загрузки, или переполнение его стакана. Момент прекращения фильтрования и начала промывки $\bar{t}_{f}$ при любом значении $\bar{\alpha}_{V}$ из предварительно выбранного диапазона легко находится из рис.4 в соответствии с (26). Анализ работы фильтра осложняется при больших значениях $\bar{\alpha}_{V}$ и активном связывании воды осажденными частицами ( $\bar{\gamma}=0,001$, пример 2). Тогда усиленное образование осадка и вызванный им быстрый рост гидравлического сопротивления загрузки обусловливают настолько серьезное снижение 
пропускной способности загрузки, что скорость $V$ начинает резко снижаться, несмотря на ускоренный подъем уровня в стакане. И тогда на первый план могут выйти экономические аспекты, а решающее значение в вышеупомянутом анализе приобрести время $t_{V}$ снижения скорости до минимально допустимого значения $V_{*}$. Впрочем, в рассмотренном случае вычисления $\bar{t}_{V}$ по (21), (28) при $\bar{V}_{*}=0,75$ (кривая 5) продемонстрировали, что хотя действительно при значениях коэффициента $\bar{\alpha}_{V}>9$ наблюдается с его увеличением падение $\bar{V}$ и, как следствие, $\bar{t}_{V}$, но для ограничения времени работы фильтра по экономическим соображениям такого падения еще недостаточно. И решающую роль здесь пока играет емкостный ресурс накопителя, который исчерпывается быстрее, чем скорость $V$ снизится до порогового значения $V_{*}$.

Итак, применительно к нисходящему фильтрованию суспензий с нерегулируемой скоростью разработан эффективный расчетный инструмент, который благодаря высокой точности может использоваться в качестве эталона при реализации упрощенных подходов, приближенных приемов решения аналогичных задач, а вместе с тем при наличии тесной связи межфазного массообмена с фильтрацией обеспечивает надежный прогноз изменений характеристик фильтрования со временем и по высоте загрузки, рациональный выбор технологических параметров и в первую очередь длительности фильтроцикла.

\section{ЦИТИРОВАННАЯ ЛИТЕРАТУРА}

1. Минц Д.М. Теоретические основы технологии очистки воды. Москва: Стройиздат, 1964. 155 с.

2. Jegftheesan V., Vigneswaran S. Deep bed filtration: mathematical models and observations. Crit. Rev. Envirom. Sci. Technol. 2005. 35(6). P.515-569.

3. Грабовский П.О. Фильтрование воды через зернистый слой с убывающей скоростью. Допов. Наи. акад. наук Укр. 2016. № 8. С. 40-45.

4. Поляков В.Л. Фильтрование суспензии с убывающей скоростью при линейной кинетике массообмена. Химия и технология воды. 2012. 34, № 2. С. 107-130.

5. Akgiray O., Saatci A.M. An algorithm for bank operation of declining rate filters. Water Res. 1998. 32, № 7. P. 2095-2105.

6. Chaundry F.H. Theory of declining rate filtration. I. Continuous operation.J. Environ. Eng. Din. ASCE. 1987. 113(4). P. 834-851.

7. Cleasby J.L. Status of declining rate filtration design. Water Sci. Technol. 1993. 27(10). P. 151-164.

8. Сенявин М.М., Веницианов Е.В., Аюкаев Р.И. О математических моделях и инженерных методах расчета процесса очистки природных вод фильтрованием. Водные ресурсы. 1977. № 2. С. 157-170.

9. Rajagopalan R., Tien C. Trajectory analysis of deep-bed filtration with the sphere-in-a-cell porous media model. AIChEJ. 1976. 2(3). P. 523-533.

10. Поляков В.Л. Осветление суспензии фильтрованием с убывающим расходом. Допов. Наи. акад. наук Укp. 2010. № 5. C. 57-63.

11. Кравчук О.А. Експериментальне дослідження витрат напору під час роботи швидкого фільтра зі змінною з часом швидкістю фільтрування. Гірничі, будівельні, дорожні та меліоративні машини. 2017. Вип. 89. С. 55-62.

12. Tobiason J.E., Vigneswaran B. Evaluation of a modified molel for deep-bed filtration. Water Res. 1994. 28. P. 335-342.

13. Поляков В.Л. Теоретический анализ длительности фильтроцикла. Химия и технология воды. 2009. 31, № 6. С. $605-618$.

Поступило в редакцию 18.07.2018

\section{REFERENCES}

1. Mints, D. M. (1964). Theoretical fundamentals of purification technology. Moscow: Stroyizdat (in Russian).

2. Jegftheesan, V. \& Vigneswaran, S. (2005). Deep bed filtration: mathematical models and observations. Crit. Rev. Envirom. Sci. Technol., 35(6), pp. 515-569. 
3. Grabovsky, P. A. (2016). Water filtration through grain layer at declining rate. Dopov. Nac. acad. nauk Ukr., No. 8, pp. 40-45 (in Russian).

4. Polyakov, V. L. (2012). Suspension filtration at declining rate and mass-exchange linear kinetics. Water Chemistry and Technology, 34, No. 2, pp. 107-130 (in Russian).

5. Akgiray, O. \& Saatci, A. M. (1998). An algorithm for bank operation of declining rate filters. Water Res., 32, No. 7, pp. 2095-2105.

6. Chaundry, F. H. (1987). Theory of declining rate filtration. I. Continuous operation. J. Environ. Eng. Din. ASCE, 113(4), pp. 834-851.

7. Cleasby, J. L. (1993). Status of declining rate filtration design. Water Sci. Technol., 27(10), pp. 151-164.

8. Seniavin, M. M., Venitsianov, E. V. \& Ayukaev, R. I. (1977). On mathematical models and engineering methods of calculating the process of natural water purification by filtratin. Water Resources, No. 2, pp. 157-170 (in Russian).

9. Rajagopalan, R. \& Tien, C. (1976). Trajectory analysis of deep-bed filtration with the sphere-in-a-cell porous media model. AIChE J., 2(3), pp. 523-533.

10. Polyakov, V. L. (2010). Susnension purification at declining rate. Dopov. Nac. acad. nauk Ukr., No. 5, pp. 57-63 (in Russian).

11. Kravchuk, A. A. (2017). Experimental investigation of head loss during rapid depth filter operation at varying filtration rate. Mining, engineering, road and melioration machines, Iss. 89, pp. 55-62 (in Ukrainian).

12. Tobiason, J. E. \& Vigneswaran, B. (1994). Evaluation of a modified molel for deep-bed filtration. Water Res., 28, pp. 335-342.

13. Polyakov, V. L. (2009). Theoretical analysis of filtration cycle duration. Water Chemistry and Technology, 31, No. 6, pp. 605-618 (in Russian).

Received 18.07.2018

\section{В.Л. Поляков}

Інститут гідромеханіки НАН України, Київ

E-mail:polyakov_igm@list.ru

\section{МОДЕЛЮВАННЯ ОСВІТЛЕННЯ СУСПЕНЗІЇ ПРИ ПОСИЛЕНОМУ ВПЛИВІ НЕРЕГУЛЬОВАНОЇ ШВИДКОСТІ ФІЛЬТРУВАННЯ НА МІЖФАЗОВИЙ МАСООБМІН}

Сформульована математична задача прояснення суспензії на швидких фільтрах з накопичувачем при нерегульованій швидкості, з якою лінійним чином пов’язані масообмінні коефіцієнти. Одержано ефективний аналітично-чисельний розв'язок задачі, який дозволяє надійно прогнозувати зміни характеристик фільтрування з часом і по висоті з врахуванням опору відвідних комунікацій, обгрунтовувати раціональні технологічні параметри і в першу чергу тривалість фільтроцикла. На характерних прикладах розв'язок ілюструється розрахунками концентрації завісі в фільтраті, швидкості фільтрування і технологічних часів.

Ключові слова: фільтрування, суспензія, нерегульована швидкість, технологія, масообмін, фільтр.

\section{V.L. Polyakov}

Institute of Hydromechanics of the NAS of Ukraine, Kiev

E-mail: polyakov_igm@list.ru

\section{MODELING THE CLARIFICATION OF A SUSPENSION WITH THE ENHANCED EFFECT OF THE UNCONTROLLED FILTRATION RATE ON THE INTERFACIAL MASS TRANSFER}

A mathematical task of the suspension purification on rapid filters with a storage device at the uncontrolled rate and a linear relation between the rate and mass exchange coefficients is formulated. An effective analytical numerical solution to the task is obtained to predict changes in filtration characteristics with regard for the hydraulic resistance of filter facilities, to substantiate rational technological parameters and, first of all, the filter cycle duration. The solution was illustrated by calculating the suspended substance concentration in a filtrate, filtration rate, and technological times.

Keywords: filtration, suspension, uncontrolled rate, technology, filter. 